\title{
La consulenza di parte nel caso Cogne
}

\author{
Elena Bianchini ${ }^{\bullet}$
}

\section{Riassunto}

L'intervista al Prof. Pietro Pietrini si focalizza principalmente sull'attività di consulenza svolta nel "caso Cogne" nei confronti di AMF. Dopo aver apprezzato i colloqui, adeguati in qualità e quantità, e il lavoro effettuato con il Prof. Balloni, consulente nominato dal Tribunale di Sorveglianza di Bologna, il Prof. Pietrini sottolinea come gli incontri avuti con AMF abbiano sicuramente rappresentato un vero e proprio inizio di un percorso psicoterapeutico che ha lo scopo di restituire la signora F. al suo ruolo di moglie, di madre, di donna al di fuori delle mura carcerarie. Infine, l'auspicio del Prof. Pietrini è che la famiglia, e in particolar modo i due figli, venga dimenticata o che quantomeno non permanga un fuoco di interesse vivo, come se il fatto fosse accaduto in tempi recenti.

\section{Résumé}

L'entretien avec M. Pietro Pietrini concerne principalement son expertise faite dans « l'affaire Cogne ».

Le Prof. Pietrini a tout d'abord exprimé sa satisfaction en ce qui concerne les entretiens avec sa cliente ; il les a trouvés appropriés en qualité et quantité. Il a aussi apprécié le travail fait en coopération avec Prof. Balloni, l'expert nommé par le Tribunal de l'application des peines (Tribunale di Sorveglianza) de Bologne.

Il précise ensuite que les rencontres avec AMF ont certainement représenté un véritable début de son parcours psychothérapeutique, dont l'objectif est de redonner à Madame F. son rôle de femme et de mère à l'extérieur des murs de la prison.

Enfin, le Prof. Pietrini espère que cette famille, en particulier les deux enfants, puisse être oubliée ou au moins que l'intérêt suscité par cette famille ne reste aussi fort que si les faits venaient de se produire.

\section{Abstract}

The interview with Prof. Pietro Pietrini is principally focused on his expert activity done in reference to the so-called "Cogne case".

First, Prof. Pietrini expressed his appreciation about the interviews carried out with his client, he finds it adequate from the point of view of both quality and quantity. He also appreciated the work done in cooperation with Prof. Balloni, the expert appointed by the Surveillance Court of Bologna.

Then, he points out that meetings with AMF have certainly represented a real beginning of her psychotherapeutic path, which aim is to give back to Lady F. her role of being a wife, a mother and a woman outside of the walls of the prison. Finally, Prof. Pietrini hopes that this family, particularly the two sons, would no longer be in the focus of attention or at least that the interest on this case will not continue since the incident happened a long time ago.

Key words: Cogne case; expert activity; psychotherapeutic path; rehabilitation of offenders; mass media.

\footnotetext{
- Dottore di ricerca in sociologia, assegnista di ricerca presso il C.I.R.Vi.S. (Centro Interdisciplinare di Ricerca sulla Vittimologia e sulla Sicurezza), Dipartimento di Sociologia e Diritto dell'Economia, Università di Bologna.
} 


\section{Introduzione.}

Mercoledì 11 febbraio 2015 mi sono recata nello studio universitario del Prof. Pietro Pietrini ${ }^{1}$ presso il Presidio Ospedaliero di Santa Chiara a Pisa. Colgo l'occasione per esprimere nuovamente la mia riconoscenza: grazie alla sua professionalità, competenza e chiarezza nell'esposizione sono emersi nuovi spunti di riflessione e punti di vista.

Sono varie le aree tematiche affrontate nel corso dell'intervista: la perizia psichiatrica, i colloqui avuti con AMF, il percorso psicoterapeutico, i mass media, il diritto della famiglia, e soprattutto dei figli, ad essere dimenticata. In particolare, il Prof. Pietrini ha sottolineato che " $i$ colloqui sono stati adeguati e numerosi" ed ha desiderato precisare che con il Prof. Balloni vi è stato "ottimo accordo sin dal primo giorno, condividendo tutto, anche nel rispetto delle diverse posizioni, nei ruoli che uno aveva". Proprio per quanto concerne i colloqui, dall'intervista emerge che "il tempo dedicato ai colloqui e soprattutto l'esperienza non comune del Prof. Balloni hanno rappresentato un vero e proprio inizio positivo di un percorso terapeutico".

Riguardo l'invasione morbosa dei mass media nelle vicende giudiziarie, l'intervistato è "dell'opinione che i processi si fanno in tribunale e le perizie si fanno nel contesto giusto" e, proprio in rapporto a questa problematica, egli afferma che "i bimbi hanno diritto ad una vita normale $e$ che quindi questi ragazzi hanno diritto ora ad essere lasciati tranquilli".

\footnotetext{
${ }^{1}$ Il Prof. Pietro Pietrini è Professore Ordinario presso il Dipartimento di Patologia Chirurgica, Medica, Molecolare dell'Area Critica, settore scientifico disciplinare: Biochimica Clinica e Biologia Molecolare Clinica.
}

\section{L'intervista' 2 .}

D: Le modalità operative del colloquio le sono sembrate adeguate e congrue in riferimento alle finalità dell'indagine? Ritiene che i colloqui abbiano avuto solo una finalità diagnostica, o che abbiano assunto anche aspetto di colloquio di sostegno e si siano inseriti nel percorso rieducativo?

R: Ritengo di sì, anche perché in realtà i colloqui sono stati molteplici, in quanto le operazioni di consulenza si sono svolte in un arco di sei mesi circa e si sono effettuati numerosi incontri. Con la signora sono stati effettuati nove o dieci incontri, comprensivi di uno al quale hanno partecipato, oltre alla signora, il marito e i figlioletti, al fine di osservare l'interazione con gli altri membri della famiglia, per vedere come si comportavano, soprattutto i figli ovviamente, cosa dicevano, come interagivano con questa mamma. Questo perché va ricordato prima di tutto che, come è stato scritto negli altri articoli che mi avete mandato, la consulenza era finalizzata a verificare la possibilità di mettere in atto da parte del tribunale una misura alternativa al carcere, cioè la detenzione domiciliare speciale, proprio al fine della tutela della salute dei figli, soprattutto il figlio minore G., il piccolo, che in effetti ha risentito molto dello stato di detenzione della mamma. Quindi, per rispondere sinteticamente, ritengo che le modalità operative del colloquio siano state adeguate e congrue. E' importante ricordare che il Prof. Balloni ed io abbiamo agito in ottimo accordo sin dal primo giorno, condividendo tutto, pur se nel rispetto delle diverse posizioni e ruoli, e abbiamo condiviso le strategie e particolari test. Con riferimento, ad 
esempio, all'esame grafologico, ammetto che non è un test al quale io avrei fatto ricorso, ma non ho avuto niente in contrario al suo utilizzo nell'ambito di questa consulenza. D'altra parte, il Prof. Balloni ha recepito molti aspetti che ho suggerito anch'io, compreso l'incontro con i familiari. I colloqui sono stati adeguati e numerosi; sulla base della mia esperienza, sono pochi i casi simili a questo per i quali le operazioni di consulenza si sviluppano attraverso tutti questi colloqui, un esame così analitico e attento della documentazione, addirittura un approfondimento chiestoci ad aprile 2014 dallo stesso tribunale. A seguito di tale richiesta, si sono svolti altri due o tre incontri, ripeto è molto difficile trovare un'attività così ricca in casi simili. Pertanto, ritengo che i colloqui siano stati adeguati sia in quantità che in qualità e abbiano altresì permesso la costruzione di un percorso nel cui ambito la condannata ha potuto esprimersi liberamente, toccando diversi aspetti della propria esistenza, fino ovviamente ai fatti tragici per cui è stata condannata. I colloqui, aspetto molto importante, hanno anche avuto l'obiettivo di guardare oltre: la signora $\mathrm{F}$. è entrata in questo percorso di consulenza in un modo e, a mio sommesso avviso, ne è uscita in un altro. A seguito dei colloqui periodici e di durata abbastanza consistente, di una visita medica, dei test, la signora ha avuto modo di riflettere su quanto emerso, di rielaborare, anche perché ad ogni colloquio si ripartiva da dove eravamo arrivati in quello precedente. Sicuramente questo può rappresentare un inizio di un percorso psicoterapeutico perché proprio noi abbiamo favorito la signora F. a pensare al dopo, a mettersi

\footnotetext{
${ }^{2}$ L'intervista è stata realizzata nello studio universitario
}

alle spalle Cogne. La signora $\mathrm{F}$. ha sempre mantenuto la sua posizione di innocenza rispetto al reato, ma ella è stata condannata dalla Cassazione, quindi siamo di fronte a due eventi inconciliabili, cioè la verità giudiziaria e la sua verità che lei ha sempre rivelato, anche in maniera coerente, identica, senza contraddizioni sin dal giorno dei fatti. Si è tenuto un processo per calunnia, il cosiddetto "Cogne bis", in cui il perito dell'accusa, il Prof. Fornari di Torino, e noi siamo giunti, da punti di partenza diversi, esattamente alla stessa conclusione. Secondo Fornari, la signora F. ha scisso e ha rimosso. Di fronte ad eventi traumatici così gravi, l'individuo fa ricorso a meccanismi psicodinamici, rimuove quella componente che sarebbe insopportabile per l'Io, cioè l'idea di avere ucciso il proprio figlio. Noi, utilizzando tecniche psico-fisiologiche come lo IAT (Implicit Association Test), cioè misurando tempi di reazione nei confronti di domande che avevano risposte vuoi nella versione accusatoria, vuoi nella versione difensiva, siamo giunti alla conclusione che la signora ha un ricordo "genuinamente innocente" della sua posizione, cioè nella mente della signora l'uccisione di S.L. non è un fatto ascrivibile alla sua volontà. Questo non significa che noi diciamo che non abbia commesso lei l'omicidio, non è compito della perizia e non è nemmeno il quesito che ci è stato posto perché non era "Cogne 1", ma era "Cogne bis". Il quesito che ci era stato posto dall' Avv. Savio e dall' Avv. Imperato, ormai qualche anno addietro, mirava a capire se nella mente di AMF l'uccisione di S.L. era un fatto ascrivibile alla sua volontà. Come abbiamo scritto nella nostra perizia, siamo giunti alla conclusione che la

del Professore e deregistrata dal supporto digitale. 
signora F. abbia un ricordo "genuinamente innocente", cioè non riconduce alla sua volontà la morte del figlio S.L. Questo va di pari passo con quanto emerso dalla perizia dell'accusa e cioè che AMF ha scisso e rimosso: si tratta di meccanismi psicodinamici di protezione dell'Io che sono inconsci, involontari, automatici e anche immediati, cioè avvengono nell'immediatezza dei fatti perché se si deve proteggere l'Io, bisogna proteggerlo prima che venga disgregato. I meccanismi di protezione dell'Io sono meccanismi che tutti noi mettiamo in atto quotidianamente, sono involontari quindi, essendo involontari, inconsci ed automatici, è chiaro che prescindono dalla volontà dell'individuo. Pertanto, anche con riferimento alla signora $F$., non si è trattato di una scelta, sono meccanismi che trascinano la volontà degli individui e come tali sono chiaramente non giudicabili. Quindi il Prof. Balloni ed io abbiamo adottato una strategia interessante ed utile soprattutto perché, ad un certo punto, abbiamo cercato di condurre i colloqui per andare oltre il caso Cogne. Occorre sottolineare, a questo proposito, che la signora F., durante i primissimi colloqui, continuava a manifestare questa sua sofferenza di persona che ritiene di non essere stata compresa, ma che in realtà ha sofferto due volte: la prima perché suo figlio è morto, la seconda perché è stata accusata e condannata per questa morte. Il continuare a professarsi innocente, e ripeto genuinamente innocente perché lei ne è fermamente convinta, come è emerso dagli accertamenti effettuati anche nel "Cogne bis", rappresenta un punto di stallo dato che, da un lato, la Cassazione ha confermato la condanna, dall'altro la condannata si professa innocente. Si potrebbe andare avanti così per altri
10 anni con la stessa sofferenza e la stessa lamentazione. Di fronte a questa impasse, noi abbiamo invitato la signora $F$. ad andare oltre: quel che è successo è successo, ora se vogliamo fare qualcosa di utile per lei e per i suoi figli, occorre che la signora F. cominci a considerare la possibilità di essere restituita al suo ruolo di moglie, di madre, di donna in una società in cui, a pena espiata, tornerà ad essere una libera cittadina. Per favorire questo percorso, abbiamo iniziato ad incentrare i colloqui proprio su tale aspetto e credo che, data la numerosità e la qualità dei colloqui, il tempo dedicato e, soprattutto, grazie anche alla non comune esperienza del Prof. Balloni, questo abbia rappresentato un vero e proprio inizio positivo di un percorso terapeutico che ha permesso, piano piano, di affrancare la signora F., di passare da un regime carcerario ad un regime dove continua a seguire un percorso di psicoterapia centrato sul suo ruolo. Pertanto, ritengo che i colloqui siano stati adeguati e soprattutto abbiano saputo fare leva su di un aspetto molto importante del futuro di AMF per uscire da questo punto di stallo. Siamo andati oltre la condizione in cui la F. è rimasta per anni, condizione che si contrappone alla realtà processuale e penso che ciò abbia rappresentato veramente un momento di sblocco e l'inizio di un nuovo percorso.

\section{D: I test mentali, al di fuori del caso in esame, le sembrano utili e necessari in questo tipo di accertamenti?}

R: Io sono uno psichiatra-biologico, un ricercatore, studio i meccanismi biochimici, fisiologici, il funzionamento celebrale, ho un'impostazione profondamente biologica, quindi non riesco a trovare nei test grafologici, ad 
esempio, quella base scientifica che mi permetta di giudicarli su dati oggettivi. Non mi sono opposto al test perché è stato suggerito dal Prof. Balloni, è un test che si utilizza da tempo, non è una sua invenzione e viene utilizzato frequentemente nell'ambito di questo tipo di accertamenti. Non riesco però a trovare in questi test indicazioni che mi permettano di concludere in un modo o in un altro. Anche io utilizzo il test grafologico, ma in quei casi in cui si debba attribuire una scrittura ad un particolare individuo, soprattutto nei casi di impugnazione dei testamenti di persone decedute. In questo senso, ritengo che la perizia grafologica sia utile perché emergono dati sull'eventuale decadimento della persona, contribuisce a fornire alcune indicazioni sullo stato cognitivo di una persona, cioè se era in grado di scrivere, se effettuava errori sintattici, se venivano manifestati cambiamenti rispetto alle capacità della persona in rapporto all'età e al grado di istruzione. In altri ambiti, invece, non ritengo che questo test sia utile, ma nel caso di specie lo è stato anche perché, avendolo somministrato proprio all'inizio delle operazioni di consulenza, è servito innanzi tutto per stabilire un contatto con questa signora, per coinvolgerla in qualcosa al fine di abbattere quel muro che sempre esiste fra esaminatore ed esaminando, era quasi "un gioco". Ritengo che il Prof. Balloni abbia scelto di avvalersi del test grafologico anche per questo motivo e occorre precisare che le decisioni non sono state prese sulla base di questo test. Generalizzando, i test che spesso vengono usati, come l'Albero di Koch, per esempio, quando vengono disposte delle CTU per stabilire le condizioni dei bambini per l'affidamento, li trovo del tutto privi di fondamento scientifico.
Indubbiamente quei test hanno il loro valore, ma devono essere usati nel modo giusto. Il disegno di un albero da parte di un bambino fornisce elementi relativamente al suo grado di maturità dato che a 3 anni lo disegna in un modo, a 5 in un altro, a 7 in un altro e così via. Se un bambino di 9 anni disegna un albero come un bambino di 3 anni, ottengo indicazioni su questo, ma non sul fatto che, dato che la chioma è spostata a sinistra, il bambino non si stacca dal passato, perché a tal proposito non c'è alcuna evidenza scientifica. $\mathrm{O}$ se la chioma è fatta a nuvoletta invece che in un altro modo è segno di chiusura verso l'esterno. E chi lo dice? Qual è l'evidenza scientifica? Ci sono lavori che dimostrano che i test proiettivi possono essere proiettati in un modo o nell'altro a seconda di chi legge; come ha detto qualcuno, $\mathrm{i}$ test proiettivi proiettano più quello che uno ci vede. Per essere sintetico, sull'uso di alcuni test ho seriamente dei dubbi. Invece, i test psicometrici, i test di personalità, sono un utilissimo completamento: il Millon, il Minnesota, ad esempio, offrono un utilissimo completamento e sottolineo completamento nel senso che la conclusione diagnostica, l'orientamento diagnostico non si tratteggia sulla base esclusiva dei risultati dei test, ma questi offrono un valido ausilio per integrare un esame psichiatrico grazie al quale si raggiunge una certa conclusione.

D: Il bisogno di conoscere le motivazioni profonde di una condotta come quelle legate al figlicidio è importante ai fini della valutazione della pericolosità sociale?

R: Indubbiamente. La pericolosità sociale è il rischio che una persona reiteri il reato per il quale è stata condannata, o se vogliamo anche una pericolosità sociale più generale nel senso del 
rischio di commettere un qualsiasi atto previsto dalla legge come reato. A proposito di ciò, insieme al Prof. Balloni abbiamo fatto un buon lavoro nel senso che abbiamo verificato appunto la sussistenza della pericolosità sociale nelle sue diverse possibili espressioni, specifica o generica. Specifica nel caso di specie, cioè qual è il rischio che la signora F. uccida un altro figlio. Noi abbiamo ritenuto che il rischio che questa signora possa uccidere un altro figlio, soprattutto il figlio più piccolo, non c'era, pur non potendo parlare in medicina del $100 \%$, cioè abbiamo considerato che non fosse significativamente rilevante dato che non esistevano elementi a supporto di quella ipotesi. Ora, se vogliamo allontanarci dal caso della signora $F$., troviamo in letteratura che una delle cause identificate nel figlicidio può essere una patologia mentale, ad esempio una depressione psicotica: ci sono mamme che uccidono figli perché ritengono di doverli proteggere dal male che può loro accadere. E' chiaro che si tratta di un'affermazione paradossale, ma va letta non nell'ambito della fisiologia, ma della patologia mentale. Il depresso grave, psicotico, uccide il proprio figlioletto per proteggerlo dai mali del mondo e spesso uccide anche se stesso, porta con sé le persone che ama, si tratta dell'omicidio-suicidio. Inoltre, una mamma può arrivare ad uccidere perché soffre di una patologia come la sindrome di Munchausen per procura, si tratta di un'altra patologia mentale, una madre può uccidere perché è schizofrenica, perché ha una psicosi per cui sente delle voci che le dicono che il figlioletto incarna il demonio. Questi sono i motivi principali. E' evidente che nessuna di queste cause si applica alla signora F. Se ci si trovasse di fronte ad una persona che è gravemente malata, che ha ucciso perché psicotica, c'è la possibilità del verificarsi di ulteriori crisi psicotiche, allora il rischio di una recidiva specifica c'è. Nella signora F. la pericolosità specifica non l'abbiamo individuata, non sono emersi elementi psicopatologici a suo carico. Bisogna considerare che l'episodio della morte di S. si è verificato il 30 gennaio 2002, noi abbiamo esaminato la signora nel 2014, quindi dodici anni dopo; in questi anni la signora è stata analizzata da molti specialisti, è in carcere da tempo, esiste una cartella clinica. Pertanto, ritengo che non ci sia alcun elemento nella storia di vita della signora $F$. che faccia pensare che ci sia stata o siano in corso manifestazioni psicopatologiche rilevanti. Infatti, è ulteriormente da precisare che la signora $F$. ha una storia psichiatrica totalmente negativa. In carcere ha manifestato qualche disturbo di adattamento, che è perfettamente fisiologico, è stata curata con una blanda terapia ansiolitica-antidepressiva, con dosaggi molto modesti, soltanto con riferimento ad un periodo le volevano somministrare qualcosa di più, ma lei non ha voluto.

In sintesi, nel corso di tutti questi anni, la signora non ha dato segni di scompenso psicopatologico, di manifestazioni psicopatologicamente rilevanti. Ella, già da tempo, ha la possibilità di lavorare fuori dal carcere nella cooperativa annessa, "Gomito a gomito", quando l'abbiamo esaminata aveva già usufruito di circa mille ore di permesso all'esterno durante le quali nulla è accaduto, quindi noi abbiamo concluso che non ci fossero elementi misurabili, in scienza e coscienza, che potessero far pensare ad una pericolosità specifica. Mi pare che il Prof. Balloni avesse parlato di una pericolosità generica nel senso di 
possibile risposta abnorme a stimoli ambientali, nel senso che la signora è stata al centro di un'attenzione mediatica senza precedenti, che si protrae ancora oggi. Dell'assedio dei giornalisti durante le udienze non ne ho fatta esperienza in nessun altro caso. Sono stato nominato perito anche in altri casi eclatanti, ad esempio quello, verificatosi nel nord dell'Italia, in cui un padre ha fatto saltare in aria la casa al cui interno vi erano due bimbetti: è stato celebrato il processo di $1^{\circ}$ grado lo scorso dicembre, fuori del tribunale si era appostata una televisione locale e nient'altro di più. Questo interesse morboso nei confronti del caso Cogne è legato a vari aspetti: al comportamento della stessa F. e della famiglia nelle prime fasi processuali, quando l'avvocato difensore non era Paola Savio, quando i periti e i consulenti non eravamo noi; a certi personaggi che hanno ruotato intorno alla famiglia e che non sono stati buoni consiglieri; anche ad un certo fascino che la signora esercita, cioè la $\mathrm{F}$. rimane nell'immaginario collettivo un mistero e, pertanto, l'Italia si è spaccata in due, tra colpevolisti e innocentisti. Questo interesse morboso è stato costante, permanente, non è mai scemato ed è arrivato ad interessare gli altri figli in maniera preoccupante. D. era un bimbo di sei-sette anni e, all'uscita della scuola, era braccato da giornalisti e fotografi: è evidente che non si trattava di una situazione piacevole.

Ho saputo di giornalisti e fotografi che sono andati ad offrire cifre non irrilevanti alle persone vicine alla famiglia per ottenere informazioni. Il piccolo S. è stato seppellito in forma anonima in uno sperduto e piccolo cimitero per evitare l'assedio mediatico. Alla signora AM sono state attribuite relazioni con Wanna Marchi, quando quest'ultima era detenuta a Bologna, commenti, relazioni, scambi di battute che, a quanto mi risulta, sono totalmente inesistenti. In sintesi, qualunque cosa potesse succedere attorno al carcere di Bologna forniva un pretesto ai mass media per parlare del caso Cogne. Allora qual è stata la questione che ci siamo posti: se una volta fuori dal carcere, non più protetta dallo schermo delle mura carcerarie, questa donna diventa il centro, il target di una persecuzione mediatica, come reagisce? Secondo il Prof. Balloni, la signora potrebbe reagire in maniera veemente nei confronti di queste pressioni, quindi si è ritenuta sussistente una pericolosità sociale nel senso di una risposta abnorme nei confronti di stimoli esterni, cosa non escludibile ovviamente. È anche vero, come ho scritto nella mia relazione, che in tutti questi anni non è mai accaduto nulla nei momenti in cui AMF era a casa, nei week-end, nessuno scatto d'ira. Quando la signora F. si accorgeva che c'era una telecamera che la puntava, anche da lontano, tornava in casa. Quindi la pericolosità generica in questo senso, a mio avviso, era un rischio estremamente modesto, conoscendo la persona e soprattutto il suo comportamento in tutti questi anni, anche in situazioni in cui era emotivamente provata, come al funerale del suocero. Tuttavia, devo dare atto che la segnalazione del Prof. Balloni, la distinzione fra pericolosità specifica e generica è molto fine, è un segno di grande esperienza ed accuratezza. Il Prof. Balloni ha ventilato il rischio di questa pericolosità ed ha proposto due modalità per arginarlo: a. con un percorso psicoterapeutico e b. soprattutto, come credo le avrà detto anche l'Avv. Savio, tramite il diritto all'oblio. Sono passati molti anni dai fatti, la signora ha espiato 
più della metà della pena, sta scontando ciò che resta in un regime alternativo, ora ha diritto all'oblio, io però temo che non sia così facile. A me è capitato in numerosissime occasioni di trovarmi in particolari contesti sociali, magari partecipando ad una cena, dove nessuno sapeva che ero il perito della F., o addirittura anche prima che lo fossi, in cui la conversazione porta a discutere di cervello, di psichiatria e spessissimo la gente comune chiede: cosa pensa lei della F.? Ovviamente, essendo io coinvolto personalmente non posso parlarne, non posso esprimere un giudizio personale, però ritengo che questo non accada con riferimento a nessun'altra vicenda giudiziaria. Tale situazione si ripercuote anche sui figli, ma per fortuna la famiglia è una famiglia sana, nel senso che il marito, in tutto questo tempo, ha saputo porsi come punto di riferimento. I familiari non hanno mai saltato una visita in carcere, nonostante ci siano limitazioni temporali, c'è stato un contatto continuo, un interesse ininterrotto. Fra l'altro, AM ha potuto avvalersi anche dell'aiuto di alcune amiche che vivono nella sua stessa zona le quali hanno contribuito a crescere G. e che, tra l'altro, sono state ritenute come uno degli elementi favorevoli per il suo percorso di reinserimento sociale. Infatti AM non è tornata a casa in un appartamento sconosciuto, ma in un paese negli Appennini che funge quasi da famiglia allargata, dove le persone vivono con le porte aperte perché i figli dell'uno mangiano a casa dell'altro. I compaesani di AM sono quelle persone che proteggono lei e la sua famiglia dal fuoco mediatico, che l'avvertono se vedono la macchina della Rai, dei volti sconosciuti armati di microfoni e telecamera o taccuino, e questo è un elemento indubbiamente favorevole. Con riferimento poi al figlio grande, è ovvio che essere il primogenito della F. sia un peso. Lei se lo immagina questo ragazzo che magari a 14-15 anni trova la ragazzina e sua mamma le chiede: con chi esci? Con il figlio della F.! Ecco provi a mettersi nei loro panni e pensare: mia figlia si è messa con il figlio della F. Questo ragazzo, quindi, subisce suo malgrado il peso, la responsabilità, si sente gli occhi addosso della gente che lo guardano come fosse un alieno, io l'ho percepito chiaramente questo. Qualsiasi cosa faccia, che per un ragazzino potrebbe essere una cosa normale, comprensibile, da adolescente, la gente potrebbe pensare che si comporta in un certo modo perché forse è come la mamma. Io non lo conosco benissimo, ma l'ho visto anche in occasione del colloquio con il Prof. Balloni e mi è sembrato un ragazzo serio, motivato, con una sua idea di lavoro e con progetti di vita. E' arrivato alla fine della scuola superiore in tempi normali, seguendo un percorso regolare, con l'idea di svolgere un lavoro tecnico, con un senso di responsabilità e un senso di pianificazione indubbiamente positivi e assolutamente non scontati.

D: Per quanto riguarda la prognosi di pericolosità sociale, è meglio attenersi a schemi statistici o occorre tenere sempre in maggior conto quelle variabili da cui dipende il comportamento, cioè la persona e l'ambiente? E' meglio dunque ricorrere alle biografie o all'uso di tabelle statistiche per quanto riguarda la prognosi di pericolosità sociale?

R: Il miglior indice predittivo del comportamento futuro è il comportamento passato. Questa è la regola principale e nel settore delle assicurazioni viene applicata regolarmente: se una persona vuole sottoscrivere un'assicurazione per la 
macchina, le chiedono quanti incidenti ha fatto; ovviamente, se risulta che lei è implicata in un incidente ogni tre mesi, non l'assicura nessuno oppure le assegnano una classe di rischio molto alta; se invece risulta che non è mai stata coinvolta in alcun incidente in venti anni di guida, la premiano. Ovviamente l'esempio non è del tutto calzante al caso in questione, tuttavia questo esempio serve per ricordare ancora una volta che la biografia è importante. Quando mi capita di periziare quelle persone che hanno un disturbo di personalità e che hanno commesso anche solo piccoli reati perché hanno un discontrollo degli impulsi, quando il giudice chiede qual è la pericolosità sociale, in questi casi si può asserire che sia veramente alta e se non c'è un intervento terapeutico, psicoterapeutico, psicofarmacologico, è chiaro che lo rifanno, è scritto nei fatti. Questo non è certamente il caso della F., però se la domanda riguarda una valutazione generale, indubbiamente è importante la biografia. È fondamentale la vita del soggetto ricostruita non solo dal soggetto, ma anche tramite documentazione oggettiva.

\section{D: I mass media possono influenzare l'opera dei consulenti?}

R: Se il perito è una persona seria, come il Prof. Balloni, no. Se il perito è una persona che cerca, ad esempio, visibilità, come nel caso di certi avvocati, allora può veramente influenzare l'operato in senso negativo. Sono dell'opinione che i processi si fanno in tribunale e le perizie si fanno nel contesto giusto, tant'è vero che io non ho mai partecipato ad alcuna trasmissione sulla F., anzi ho declinato più di un invito. Se il perito è serio, i mass media influenzano zero, come deve essere. Se il perito non è serio allora non è un perito, non è un consulente, allora fa spettacolo. Il perito deve lavorare sui dati, sui fatti, senza lasciarsi influenzare. Tutti siamo influenzabili in maniera inconscia, ovviamente, però dobbiamo lavorare senza lasciarci influenzare. Per fare questo occorre lavorare senza fretta. La perizia $\mathrm{F}$. è stata faticosissima perché ci siamo visti 11 o 12 volte, il materiale da studiare era tanto, insomma questa è una perizia seria. Poi si può anche ritenere che ad impossibilia nemo tenetur, quindi nessuno può fare le cose impossibili, però noi abbiamo fatto il massimo nel miglior modo possibile per le nostre conoscenze.

Un altro aspetto di base è fondamentale: il caso F. è un caso particolare in relazione agli incidenti domestici. Cosa intendo dire? Prima di rispondere a questa domanda, porto un esempio personale. Sulla fronte ho questa cicatrice che mi sono provocato quando avevo 7 anni perché, correndo in casa per andare in bagno a fare la doccia dopo una giornata trascorsa al mare, sono scivolato e ho battuto la testa contro la porta. Mio padre mi portò al pronto soccorso e i medici mi curarono perché avevo la testa spaccata. Si immagini cosa potrebbe succedere se arrivasse G. al pronto soccorso con la testa spaccata. Chi lo spiegherebbe ai medici che la ferita è stata provocata da una caduta accidentale mentre correva per andare in bagno? Chi ci crederebbe? Queste cose, invece, possono accadere. Tutti noi abbiamo almeno una storia da raccontare relativamente a bambini che si sono feriti a causa di banali incidenti domestici, lei si immagini lo stesso incidente domestico di cui rimane vittima il figlio minore di AF.... Allora è ovvio che noi consulenti abbiamo dovuto ben documentare il fatto di aver esplorato tutti i meandri di quella mente, del comportamento e 
della storia della signora in modo tale da poter ritenere che i pericoli di commissione di un'azione volontaria siano estremamente bassi, se non nulli.

\section{D: Nei casi di figlicidio, nel percorso} rieducativo, quali supporti dovrebbero essere forniti alle madri che si macchiano di tale delitto?

$\mathrm{R}$ : In generale, quindi uscendo dal caso specifico della signora F., il percorso riabilitativo deve essere centrato sulla persona e si deve partire dalla domanda: perché questa persona ha ucciso il figlio? Se è una persona che soffre di una patologia depressiva grave, l'intervento dovrà consistere nel tentativo di tenere sotto controllo la situazione, riequilibrare con interventi anche clinicamente importanti, ricovero, psicoterapia, psicofarmacologia, terapia farmacologica, cercare di mitigare, non voglio dire risolvere perché in psichiatria come in medicina risolvere è una parola molto impegnativa, ma tenere sotto controllo, ripristinare un equilibrio psico-fisico, soprattutto psichico, adeguato e mettere in atto quelle misure che possano contenere eventuali nuove alterazioni del comportamento. Pertanto, l'intervento dipende dal motivo che lo ha generato e se il motivo è identificabile: può essersi verificato uno stato di psicosi acuta, la persona soffre di sindrome di Munchausen, il reato è stato un reato di impeto, si è verificato un discontrollo degli impulsi, ecc..

Desidero sottolineare che io, in questo caso, ho assunto il ruolo di consulente di parte, ma veramente l'azione è stata concertata e portata avanti congiuntamente e in maniera coordinata. Ho un ricordo molto piacevole del lavoro svolto.

Dato che la signora F. ha mantenuto fermo il suo atteggiamento nel professarsi assolutamente innocente e manifestando anche questa notevole sofferenza e lamentazione, il meccanismo chiave del nostro lavoro è stato quello di andare oltre. Nel caso di specie, dunque, secondo noi, la svolta da imprimere consisteva nell'andare oltre, guardare al futuro, quindi lavorare per riuscire a restituire alla F. la dignità, la capacità di essere donna nella società, donna nella famiglia, moglie e mamma. Quindi è questo il vero percorso, quello che è stato è stato, anche aderendo alla tesi della F. che si dichiara innocente. Ci sono sicuramente degli errori giudiziari e da scienziato, da ricercatore, posso dire che gli errori giudiziari esistono. A tal proposito, porto l'esempio di un progetto americano, il Freedom project, nel cui ambito il test del DNA, che è diventato disponibile successivamente, ha dimostrato che $\mathrm{i}$ 228 condannati detenuti in quel momento, rei confessi, condannati anche per reati gravi, pure persone nel braccio della morte, non c'entravano nulla. Perciò, se c'è un reo confesso che però non è il vero reo vuol dire che si innescano meccanismi talmente complessi, anche nella psiche umana, che devono far riflettere.

Ciò premesso, qual è stata la nostra opera: lasciamo perdere tutte queste cose, "io $\mathrm{mi}$ professo innocente", "io voglio giustizia", "combatterò sino all'ultimo giorno", perché se tu combatti sino all'ultimo giorno è una fonte di sofferenza, è una lotta contro i mulini a vento, a meno che non salti fuori, come è accaduto in molti casi, la persona che in punto di morte confessa. Ma a meno che non succeda una cosa del genere, mantenere una posizione di principio non giova. Ripeto, la F. è genuinamente convinta di essere innocente: la verità processuale e la realtà che uno vede sono due cose diverse; tra avere il mal di 
pancia ed essere convinti di avere il mal di pancia non c'è differenza, se uno è convinto di avere mal di pancia e uno ha mal di pancia perché ha qualcosa dentro la pancia non c'è differenza soggettivamente, quindi per lei non c'è differenza. Il percorso psicoterapeutico nel caso di specie è stato da noi orientato, e continua ad essere orientato, nell'andare avanti, nel non continuare a rivangare il passato perché, così facendo, si rischia di rimane fermi, fossilizzati e questo è contro evolutivo per l'individuo dato che l'individuo ha bisogno di andare oltre. Il percorso terapeutico è stato improntato in questa direzione, abbiamo preso per mano la signora $F$. e, pian piano, l'abbiamo portata a vedere le cose in maniera diversa da come le vedeva il giorno del primo colloquio in cui ha mostrato in pieno tutta la sua sofferenza, tutto il suo lamentio anche per non essere stata mai compresa come mamma che ha perso il bambino. Alla fine ha però assunto una posizione diversa, dove non si guardava indietro o nell'hic et nunc, cioè nello status quo, ma si guardava avanti, perché ci sono prospettive future: questo è quello che va fatto. Tutta questa opera che abbiamo fatto il Prof. Balloni ed io non era finalizzata a mandare a casa la $\mathrm{F}$. per farle un favore, ma era valutare se la signora $F$. potesse andare a casa nell'interesse del figlio minore G., che è un bambino che stava soffrendo molto per la lontananza dalla mamma e, paradossalmente, stava soffrendo di più negli ultimi tempi rispetto a prima, per due ragioni: perché prima era piccolo e i bambini piccoli hanno dei meccanismi di difesa enormi, potentissimi; perché la mamma era comunque in una situazione cristallizzata, la mamma è là, la mamma sta bene, io so che la mamma sta bene, non me ne preoccupo. Nel momento in cui la mamma ha cominciato ad arrivare a casa ogni quindici giorni, questo è stato emotivamente destruente per G. e lo possiamo facilmente immaginare: il venerdì arriva la mamma, quindi c'è la gioia della presenza della mamma, e la domenica sera o il lunedì mattina la mamma se ne va di nuovo, e io aspetto altri 15 giorni. Per un bambino di 9-10 anni è difficile potersi spiegare perché è così e allora G. metteva in atto dei meccanismi che devono spiegare il perché. E lì si è innescato un meccanismo psicologicamente e scientificamente molto interessante, molto bello, ma anche molto doloroso per il bambino: la mamma non è qui perché c'è la nonna, le due sono mutualmente esclusive e il bambino ha dovuto razionalizzare, concretizzare, esprimere, incanalare la sua sofferenza dicendo che la colpa del fatto che non c'è la mamma qui è della nonna, quindi se la nonna se ne va la mamma torna, questo è il pensiero concreto del bambino; cioè se quando c'è la mamma la nonna non c'è e quando c'è la mamma non c'è la nonna, vuol dire che se la nonna se ne va allora torna la mamma. E quindi è la nonna la causa, è la nonna la colpa di tutta la mia sofferenza e la rabbia la indirizzo verso la nonna. Finché lui era piccolo e finché la mamma era in carcere, la vedeva sei ore al mese, la situazione era cristallizzata, la mamma era protetta dalle mura, sta bene. Secondo me, AM. è stata bravissima in questo perché non ha mai fatto trasparire la propria sofferenza nei confronti dei figli, è sempre stata una donna forte e questo è un ulteriore aspetto che abbiamo valutato. Nel momento in cui la mamma arriva, mi accompagna a scuola e poi non la vedo più, è stato devastante ed io ho detto e scritto che, paradossalmente, 
sarebbe meglio che questa donna a casa non ci andasse per nulla. Il Prof. Balloni ed io abbiamo punti di vista diversi, prospettive differenti, ma sostanzialmente si guardava nella stessa direzione. Il Prof. Balloni ed io ci siamo trovati d'accordo, se c'è stata qualche differenza è perché uno guardava da destra verso sinistra e uno da sinistra verso destra, ma si stava descrivendo esattamente la stessa cosa. E poi ci sono ovviamente anche i ruoli diversi, però dicevamo sostanzialmente la stessa cosa. I giornali l'hanno riportata in maniera diversa, sembra che AM. fosse stata premiata e mandata a casa, ma è bene sottolineare che questo provvedimento serve a tutelare gli interessi del bambino. Questa famiglia si mette alla prova con le difficoltà che hanno le famiglie tutti i giorni, con un passato che deve essere assolutamente superato e finalmente consegnato all'oblio. Per loro non sarà mai un oblio, per loro questo rimarrà sempre, però non si può nemmeno pensare che questa signora venga additata mentre cammina per strada o si formino capannelli, o ci siano giornalisti che dicono che la F. si è comprata un gelato. Speriamo che venga dimenticata o che venga ricordata come deve essere ricordata, ma non che permanga un fuoco di interesse vivo come se il fatto fosse accaduto questa settimana. Se chiede a 100 persone chi è la F., 100 persone le rispondono e le danno anche una loro versione, le loro spiegazioni e questo non può più essere così, soprattutto nell'interesse dei figli. C'è stato un interesse morboso, con giornalisti e fotografi che cercavano di ottenere informazioni offrendo denaro agli amici, ai vicini di casa, cifre pari a 2 , 3,4 mesi di stipendio. Il bimbo è sepolto in un luogo che nessuno sa perché altrimenti diventa meta di curiosità, di foto. Il bambino piccolo era assediato all'uscita della scuola, del resto non dico nulla, basta che guardi su Internet. Io definisco tutta questa situazione come pornografia emotiva, per questi bimbi soprattutto, lei e il marito sono due adulti, ma i bimbi hanno diritto ad una vita normale, che già non avranno a causa della situazione perché sono bimbi cresciuti con la mamma in galera, accusata e condannata per aver ucciso il loro fratellino, sradicati dalla loro casa, quindi parlare di vita normale è impossibile. Come ha detto l'Avv. Savio, che tra l'altro ha condotto le cose benissimo, i bambini hanno diritto all'oblio ed è segno di quanto la famiglia e gli altri siano stati in grado di fare da punto di riferimento, soprattutto il padre perché è un miracolo che D. sia un ragazzo essenzialmente normale, più normale di molti altri. Quindi questi ragazzi hanno diritto ora ad essere lasciati tranquilli.

\section{Conclusioni.}

Il Prof. Pietrini in questa intervista ha dunque ben sottolineato due concetti: che la misura alternativa alla detenzione della detenzione domiciliare speciale viene elargita nell'interesse dei figli minori, e non della condannata, e che il percorso rieducativo debba essere improntato nel lasciarsi alle spalle ciò che purtroppo è successo e di guardare avanti.

Per quanto concerne le misure alternative alla detenzione, è utile rilevare che alla normale espiazione della pena detentiva si affiancano altre modalità di punizione e tali misure costituiscono le sanzioni penali che devono dare attuazione al principio dell'art. 27 della Costituzione italiana secondo cui le pene devono tendere alla rieducazione del condannato. Infatti, "presupposto 
fondamentale per la concessione di forme di esecuzione della pena alternative al carcere è la idoneità della misura alternativa a contenere il pericolo di recidiva e favorire il percorso di risocializzazione",3. Attraverso il sistema delle misure alternative viene realizzato il principio di flessibilità dell' esecuzione penale, che risponde ad esigenze sia umanitarie che solidaristiche. Come esito di questo principio, la pena non costituisce più un dato fisso e immutabile, ma si può modificare in conseguenza delle condizioni soggettive ed oggettive previste dalla legge, oltre dal fatto che la quantità e la qualità di pena espiata abbia assolto o meno il fine rieducativo ${ }^{4}$.

Per quanto concerne nello specifico la detenzione domiciliare speciale, il Prof. Pietrini sottolinea "prima di tutto che [...] la consulenza era finalizzata a verificare la possibilità di mettere in atto da parte del tribunale una misura alternativa al carcere, cioè la detenzione domiciliare speciale, proprio al fine della tutela della salute dei figli minori, soprattutto il figlio minore G., il piccolo, che in effetti ha risentito molto dello stato di detenzione della mamma". In un altro punto dell'intervista, il consulente di parte ribadisce ancora questo concetto: "i giornali l'hanno riportata in maniera diversa, sembra che AM. fosse stata premiata e mandata a casa, è bene sottolineare che questo provvedimento serva a tutelare gli interessi del bambino" minore che ha particolarmente sofferto di questa situazione, soprattutto quando sono intervenuti i permessi premio concessi alla condannata ogni quindici

3 Fiorentin F., Marcheselli A., Il giudice di sorveglianza: la giurisprudenza dei tribunali e dei magistrati di sorveglianza, Milano, Giuffrè, 2008.

${ }^{4}$ Degl'Innocenti L., Faldi F., Misure alternative alla detenzione e procedimento di sorveglianza, Giuffrè, Milano, 2006. giorni: "finché lui era piccolo e finché la mamma era in carcere, la vedeva sei ore al mese, la situazione era cristallizzata, la mamma era protetta dalle mura, sta bene. Secondo me, AM. è stata bravissima in questo perché non ha mai fatto trasparire la propria sofferenza nei confronti dei figli, è sempre stata una donna forte e questo è un ulteriore aspetto che abbiamo valutato. Nel momento in cui la mamma arriva, mi accompagna a scuola e poi non la vedo più, è stato devastante ed io ho detto e scritto che, paradossalmente, sarebbe meglio che questa donna a casa non ci andasse per nulla".

La famiglia è quindi il nucleo che deve essere preservato e rinforzato, tenendo in considerazione che l'esecuzione della pena "riflette ineluttabilmente ed oggettivamente i suoi effetti non solo sulla vita del condannato, ma anche su quella del coniuge, dei figli, dei familiari e conviventi" istituzioni totali sono incompatibili anche $[\ldots]$ con la famiglia"6. Una tematica particolarmente dolorosa riguarda quindi il mantenimento delle relazioni parentali e amicali, che si concretizzano in scambi epistolari e in brevi visite alla presenza di terzi estranei, ad esempio le forze dell'ordine o gli assistenti sociali. Non bisogna pertanto ignorare le reali ed immediate difficoltà che un condannato si trova ad affrontare, nel momento in cui viene condannato, nei confronti della famiglia, dei colleghi, degli amici, dei vicini di casa, che si ripercuotono anche nel momento in cui una persona esce dalle mura carcerarie per affrontare

\footnotetext{
${ }^{5}$ Mastropasqua G., Esecuzione della pena detentiva $e$ tutela dei rapporti familiari e di convivenza. I legami affettivi alla prova del carcere, Cacucci, Bari, 2007, p. 21 .
} 
una diversa modalità di espiazione della pena. Si tratta infatti di una "famiglia che si mette alla prova con le difficoltà che hanno le famiglie tutti $i$ giorni, con un passato che deve essere assolutamente superato e finalmente consegnato all'oblio. Per loro non sarà mai un oblio, per loro questo rimarrà sempre, però non si può nemmeno pensare che questa signora venga additata mentre cammina per strada o si formino capannelli, o ci siano giornalisti che dicono che la F. si è comprata un gelato". L'interesse dei mass media è stato talmente invadente da essere definito "pornografia emotiva, per questi bimbi soprattutto, lei e il marito sono due adulti, ma $i$ bimbi hanno diritto ad una vita normale, che già non avranno a causa della situazione perché sono bimbi cresciuti con la mamma in galera, accusata e condannata per aver ucciso il loro fratellino, sradicati dalla loro casa", invocando ancora una volta, come l'Avv. Savio e il Prof. Balloni, il diritto di questi ragazzi "ora ad essere lasciati tranquilli".

Alla domanda se questa invasione dei mass media potesse influenzare la professionalità di un consulente, il Prof. Pietrini ha risposto negativamente: "se il perito è una persona seria, come il Prof. Balloni, no. Se il perito è una persona che cerca, ad esempio, visibilità, come nel caso di certi avvocati, allora può veramente influenzare l'operato in senso negativo. Sono dell'opinione che i processi si fanno in tribunale e le perizie si fanno nel contesto giusto, tant'è vero che io non ho mai partecipato ad alcuna trasmissione sulla $F$., anzi ho declinato più di un invito. Se il perito è serio, $i$ mass media

${ }^{6}$ Goffman E., Asylums. Le istituzioni locali: $i$ meccanismi dell'esclusione e della violenza, Piccola Biblioteca Einaudi, Torino, 2010, p. 41. influenzano zero, come deve essere. Se il perito non è serio allora non è un perito, non è un consulente, allora fa spettacolo".

Il prof. Pietrini non esita a sottolineare che "tutti siamo influenzabili in maniera inconscia, ovviamente, però dobbiamo lavorare senza lasciarci influenzare. Per fare questo occorre lavorare senza fretta. La perizia $F$. è stata faticosissima perché ci siamo visti 11 o 12 volte, il materiale da studiare era tanto, insomma questa è una perizia seria. Poi si può anche ritenere che ad impossibilia nemo tenetur, quindi nessuno può fare le cose impossibili, però noi abbiamo fatto il massimo nel miglior modo possibile per le nostre conoscenze".

L'assedio mediatico e l'interesse morboso nei confronti degli avvenimenti che vedono come protagonista AMF è stato "costante, permanente, non è mai scemato" ed è stato inoltre preso in considerazione quando i consulenti si sono dovuti esprimere sulla pericolosità sociale generica della condannata: "allora qual è stata la questione che ci siamo posti: se una volta fuori, non più protetta dallo schermo delle mura carcerarie, questa donna diventa il centro, il target di una persecuzione mediatica, come reagisce? E il Prof. Balloni ha detto: potrebbe reagire in maniera più, non dico violenta, ma veemente, a queste pressioni. Quindi c'era una pericolosità sociale nel senso di una risposta abnorme nei confronti di stimoli esterni, cosa non escludibile ovviamente. $\grave{E}$ anche vero, come ho scritto io nella mia relazione, che in tutti questi anni questo non è mai accaduto".

L'altra questione di rilevante importanza che è stata sollevata nell'intervista riguarda il percorso riabilitativo che AMF dovrà affrontare. I colloqui 
intervenuti con la condannata sono "stati adeguati sia in quantità che in qualità $e$ hanno altresì permesso la costruzione di un percorso nel cui ambito la condannata ha potuto esprimersi liberamente, toccando diversi aspetti della propria esistenza, fino ovviamente ai fatti tragici per cui è stata condannata", rappresentano sicuramente "un inizio di un percorso psicoterapeutico, perché proprio noi abbiamo favorito la signora $F$. a pensare al dopo, a mettersi alle spalle Cogne [...] : quel che è successo è successo, ora se vogliamo fare qualcosa di utile per lei, per i figli, cioè se vogliamo che questo avvenga, bisogna che la signora F. entri nell'ottica di considerare la possibilità di essere restituita al suo ruolo di moglie, di madre, di donna in una società dove a pena espiata, cioè una volta che abbia completato di espiare la pena alla quale è stata condannata, quindi anche in forma di regime di detenzione domiciliare, poi tornerà ad essere, come tutti $i$ condannati, una libera cittadina".

Questa situazione di empasse può dunque essere superata impostando un percorso psicoterapeutico che sia orientato all'andare avanti, non al "rivangare il passato perché, così facendo, si rischia di rimane fermi, fossilizzati e questo è contro evolutivo per l'individuo dato che l'individuo ha bisogno di andare oltre".

\section{Bibliografia,}

- Balloni A., Criminologia e psicopatologia. Analisi di 110 perizie psichiatriche, Patron, Bologna, 2004.

- Balloni A., Bisi R., Sette R. (a cura di), Manuale di criminologia - I. Le teorie, Clueb, Bologna, 2013.

- Balloni A., Bisi R., Sette R. (a cura di), Manuale di criminologia - II. Criminalità, controllo, sicurezza, Clueb, Bologna, 2013.

- Bianchini E., La riabilitazione tra estinzione del reato e riparazione alla vittima. La sua applicazione in Italia $e$ in Belgio, Edizioni Accademiche Italiane, Saarbrüken, 2013.

- Bianchini E., "Il trattamento penitenziario del condannato", in Balloni A., Bisi R. Sette R., Manuale di criminologia - II. Criminalità, controllo, sicurezza, Clueb, Bologna, 2013, pp. 283-301.

- Bisi R., "Giustizia, carcere, riabilitazione del criminale e solidarietà sociale", in Balloni A., Bisi R., Sette R. (a cura di), Manuale di criminologia - II. Criminalità, controllo, sicurezza, Clueb, Bologna, 2013, pp. 273-281.

- Degl'Innocenti L., Faldi F., Misure alternative alla detenzione e procedimento di sorveglianza, Giuffrè, Milano, 2006.

- Fiorentin F., Marcheselli A., Il giudice di sorveglianza: la giurisprudenza dei tribunali $e$ dei magistrati di sorveglianza, Milano, Giuffrè, 2008.

- Goffman E., Asylums. Le istituzioni locali: $i$ meccanismi dell'esclusione e della violenza, Piccola Biblioteca Einaudi, Torino, 2010.

- Mastropasqua G., Esecuzione della pena detentiva e tutela dei rapporti familiari e di convivenza. I legami affettivi alla prova del carcere, Cacucci, Bari, 2007. 